\title{
Selected cases of heat transfer phenomena on the shock waves in atmospheric air
}

\author{
Andrzej Wilk ${ }^{1, *}$, and Stawomir Dykas ${ }^{1}$ \\ ${ }^{1}$ Silesian University of Technology (STU), Institute of Power Engineering and Turbomachinery, \\ 44-100 Gliwice, Konarskiego 18, Poland
}

\begin{abstract}
The content of water vapour, liquid water or ice in a dispersed form in the atmospheric air is very common and it might affect the aerodynamic characteristics, especially in the transonic or supersonic flow regime. In the paper, special attention was paid to identifying the heat transfer phenomena appearing on a transition from sub- to supersonic, and vice versa flow regime. The in-house CFD code was employed for performing the numerical analysis. The CFD calculations were carried out for the geometry of the straight channel with a bump as well as blade-to-blade channel for different boundary conditions, ratio of outlet static pressure to inlet total pressure. The numerical results showed a clear dependence of the sonic region and wetness mass fraction formation.
\end{abstract}

\section{Introduction}

Employment of the experimental techniques made significant progress in the research on the transonic flows of atmospheric air. For example, in 1935 at aerodynamics congress Prandtl presented a Schlieren picture of two crossing oblique shock waves near the throat in a nozzle flow with no back pressure. It was concluded that this structure is dependent on the initial humidity of the air. This disturbance was called a condensation shock wave and for the nozzle flow it has the X-shape structure. In the 90 s of former century Schnerr has done CFD calculations on different types of supersonic nozzles, confirming numerically presence of the X-shape condensation wave in nozzle flows $[5,6]$. The process of water vapour condensation in the atmospheric air flow is still valid and deals with both external [7] as well as internal flows [8].

In internal flows with fast expansion the development of the liquid phase is dominated by homogeneous condensation. However, in case of the impurities presence in air the heterogeneous condensation may take part as well, and by high concentration of the solid particles it may have dominant character [3]. The process of liquid phase formation undergoes abruptly producing significant losses in the flow. If the Mach number is in the transonic regime, the flow is near the maximum local mass flow rate and the timescale of cooling is in the range of the timescale of the agglomeration of the vapour molecules $(\sim 1 \mu \mathrm{s})$, hence the flow has enough energy to release the heat (latent heat) for condensing the steam comprised in the air to satisfied the energy equation [1].
In this paper the numerical analysis of the homogeneous condensation process in the vicinity of the sonic flow regime was presented for two geometries and for different flow conditions. For all test cases at the inlet the relative humidity of $70 \%$ was assumed, whereas the flow conditions were changed by decreasing the outlet static pressure at the investigated geometry outlet.

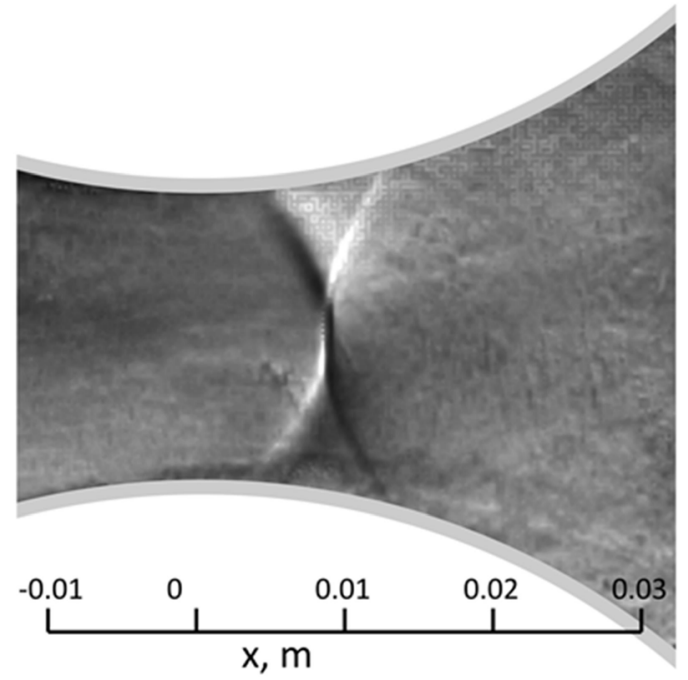

Fig. 1. Schlieren image obtained experimentally at SUT for arc nozzle with air humidity $\sim 70 \%[2]$

\section{PHYSICAL MODEL}

The CFD calculations presented herein were made by means of an in-house academic code, which has been developed and used for many years for many engineering applications, including the modeling of moist air flows [1, $2,3]$.

Corresponding author: andrzej.t.wilk@polsl.pl 


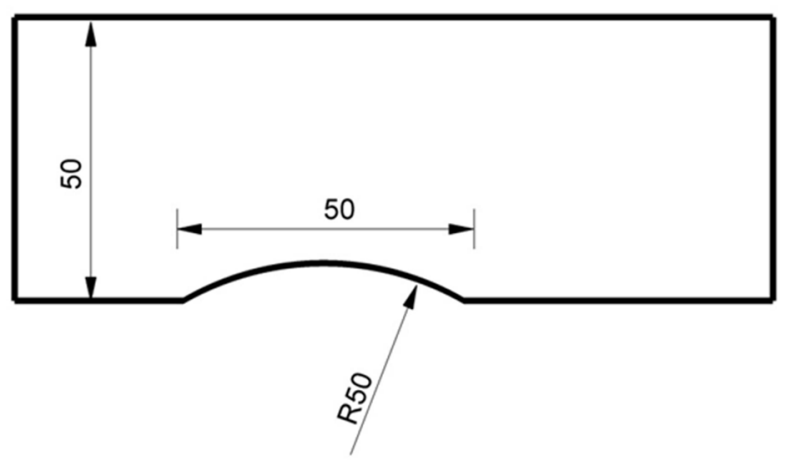

Fig. 2. Analyzed geometry of the channel with a bump (main dimensions in millimeters)

The applied computational code was mainly used to identify the condensation phenomenon in the transonic flow. The presented code solves the RANS equations by means of the third-order MUSCL-type TVD finite-volume scheme with the explicit second-order Runge-Kutta method for time integration.
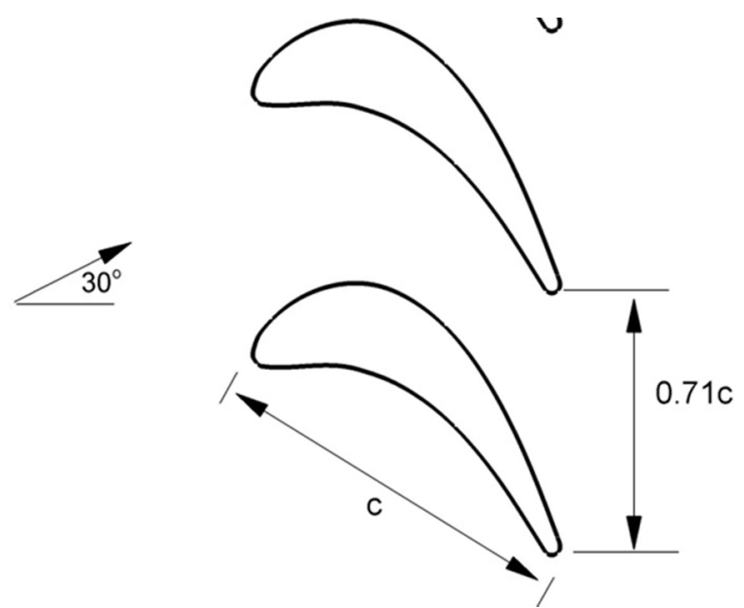

Fig. 3.Geometry of turbine blades cascade [4]

The finite-volume cell-centered discretization for a multi-block structured mesh was employed. The k- $\omega$ Shear Stress Transport viscous turbulence model was applied. The used model of the compressible, viscous and turbulent flow of moist air is composed of:

- the mass, momentum and energy conservation equations for the air and steam/water mixture,

- the turbulence model equations,

- the transport equations for the liquid phase arising due to homogeneous condensation,

- the relations modeling the condensation process according to the classical theory of nucleation and to the molecular-kinetic droplet growth model,

- the ideal gas equation of state.

\section{RESULTS AND CONCLUSIONS}

As it was previously mentioned, for the nozzle flow of moist air the homogenous condensation process become visible in form of X-shape wave (Fig. 1). Whereas, for the other geometries, especially non-symmetric one, it is not clear to identify the condensation process on the basis of the Schlieren photographs.
In the case of CFD modeling the best way is to identify the onset of the wetness mass fraction.

For the CFD analysis the geometries of the straight channel with a bump (Fig. 2) as well as blade-to-blade channel (Fig. 3) were assumed. In inlet total parameters were 1 bar $_{a}$ and $18^{\circ} \mathrm{C}$, the inlet relative humidity was set to $70 \%$ for all cases. For each geometry three flow conditions were considered, corresponding the ratio of outlet static pressure to total inlet pressure $0.7,0.6$ and 0.5 . These pressure ratios correspond to the different range of flow regime, the lower pressure ratio the higher Mach number and the wider transonic flow region should be.

The numerical meshes assumed in the CFD calculations ensured the grid independent solution, $\mathrm{y}+$ was for both geometries less than 2 .

In the Figures 4-6 the wetness mass fraction distributions for both geometries were presented, together with marked sonic lines $(\mathrm{Ma}=1)$. The range of presented liquid mass fraction was $0 \div 8 \mathrm{~g}_{\text {water }} / \mathrm{kg}_{\text {air }}$ with the step $1 \mathrm{~g}_{\text {water }} / \mathrm{kg}_{\text {air }}$.

It is visible that the condensation onset is closely related to transonic flow regime in the flow. Also, the value and the area of occurrence of wetness mass fraction is affected by the transonic flow regime. The wetness mass fraction quantity may decrease on the transition from supersonic to subsonic flow region in the result of partial evaporation.
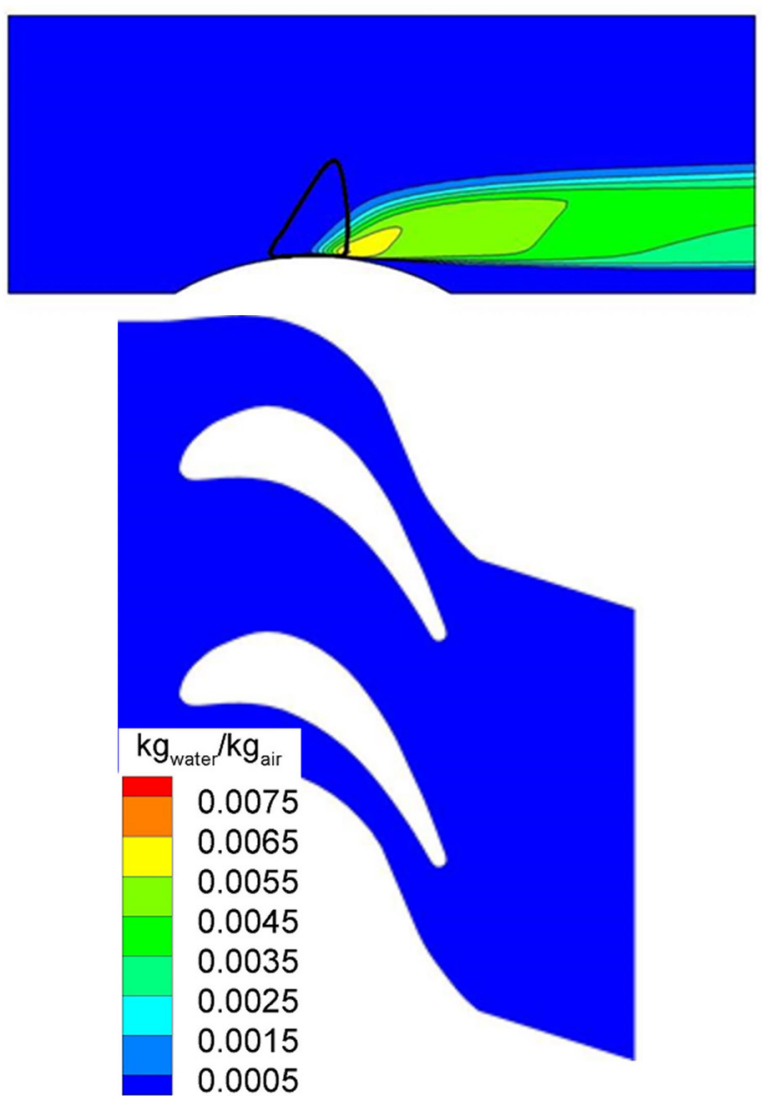

Fig. 4. Sonic lines $(\mathrm{Ma}=1)$, bold solid line and wetness mass fraction distribution for pressure ratio 0.7 (upper: channel with a bump; lower: turbine blades cascade) 

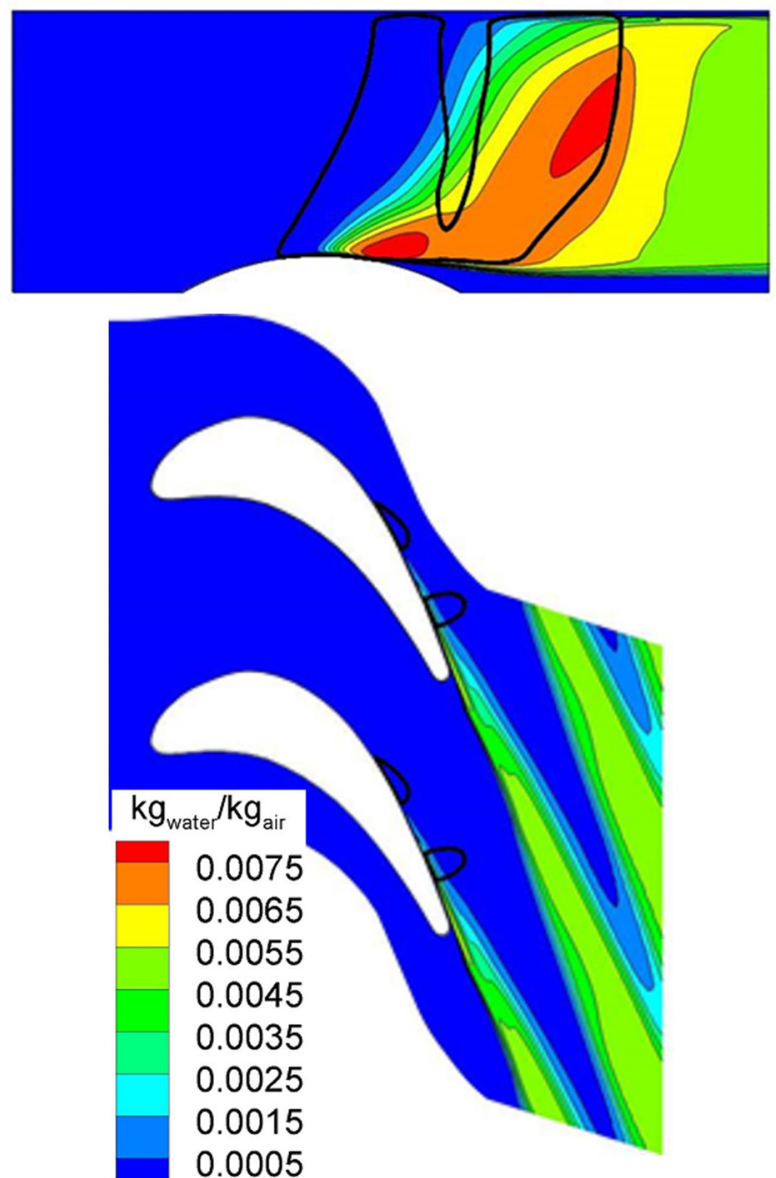

Fig. 5. Sonic lines $(\mathrm{Ma}=1)$, bold solid line and wetness mass fraction distribution for pressure ratio 0.6 (upper: channel with a bump; lower: turbine blades cascade)

For the pressure ratio above the critical value, which for the ideal gas amounts to 0.528 , the transonic flow regime is located in the places of local flow acceleration, i.e. on the bump or on the suction side of the blade close to the trailing edge. Whereas, for the pressure ration less than 0.528 the transonic flow region covers almost entire outlet area.

However, for pressure ratio 0.7 for blade cascade there is no wetness mass fraction (Fig. 4 lower), and the reason for this is no transonic regime in the flow (Fig. 7). It means that the flow has no enough energy to precipitate the liquid phase due to the spontaneous condensation.

Publication supported as a part of the Rector's grant in the area of scientific research and development works, Silesian University of Technology (STU), Poland, grant number RGJ 08/050/RGJ18/0152.
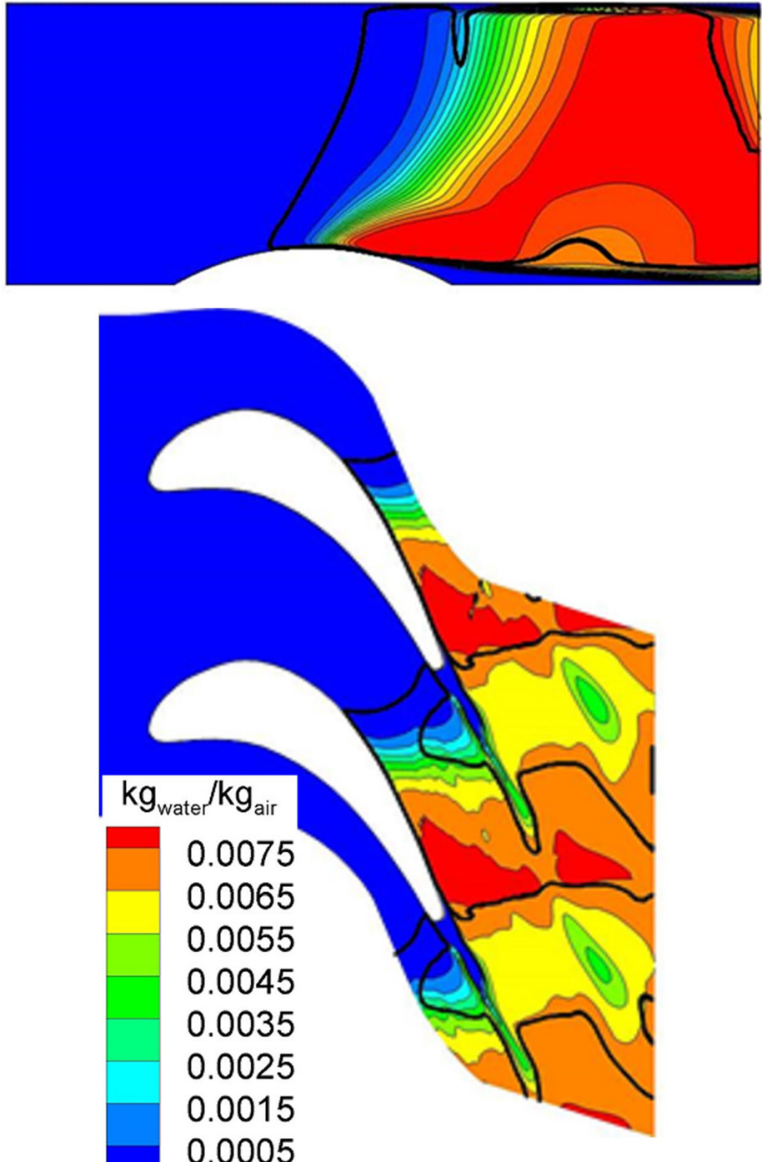

Fig. 6. Sonic lines $(\mathrm{Ma}=1)$, bold solid line and wetness mass fraction distribution for pressure ratio 0.5 (upper: channel with a bump; lower: turbine blades cascade)

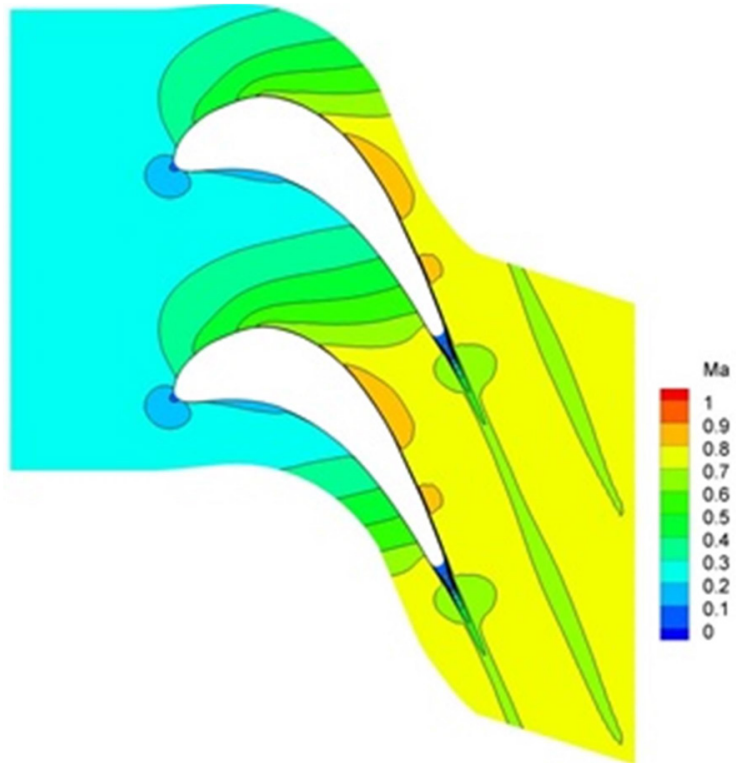

Fig. 7. Mach number distribution for blade cascade for pressure ration 0.7 


\section{References}

1. P. Doerffer, S. Dykas S., Numerical analysis of shock induced separation delay by air humidity, Journal of Thermal Science 14 (2), pp. 120-125, (2005)

2. S. Dykas, M. Majkut, K. Smołka, M. Strozik, Comprehensive investigations into thermal and flow phenomena occurring in the atmospheric air twophase flow through nozzles, International Journal of Heat and Mass Transfer, 114, p. 1072-1085, (2017)

3. S. Dykas, M. Majkut, K. Smołka, M. Strozik, Numerical analysis of the impact of pollutants on water vapour condensation in atmospheric air transonic flows, Applied Mathematics and Computation, 338, p. 451-465, (2018)

4. R. Kiock, F. Lehthaus, N. Baines, C. Sieverding, The Transonic Flow Through a Plane Turbine Cascade as Measured in Four European Wind Tunnels. J. of Eng. for Gas Turbine and Power, vol. 108, p. 277-287, (1986)

5. G. Schnerr, G. Mundinger, Similarity, drag, and lift in transonic flow with given internal heat addition, Euro. J. Mech., B/Fluids, 12 (5), p. 597-611, (1993)

6. G. Schnerr, U. Dohrmann, Transonic flow around airfoils with relaxation and energy supply by homogeneous condensation, AIAA Journal, 28, p. 1187-1193, (1990)

7. S. Yamamoto, H. Hagari, M. Murayama, Numerical simulation of condensation around the 3-D Wing, Transactions of the Japan Society for Aeronautical and Space Sciences, 42 (138), p. 182-189, (2000)

8. S. Yamamoto, S. Moriguchi, H. Miyazawa, T. Furusawa, Effect of inlet wetness on transonic wetsteam and moist-air flows in turbomachinery, International Journal of Heat and Mass Transfer, 119, p. 720-732, (2018) 